\title{
Mola hidatiforme parcial e completa: características clínicas e histológicas
}

\section{Complete and partial hydatidiform mole: clinical and histological presentation}

Larissa Oliveira Galindo Almeida ${ }^{1}$. Edson de Macedo Sousa ${ }^{1}$. Ana Carolina Montes Ribeiro ${ }^{1}$. Diane Isabelle Magno Cavalcante ${ }^{1}$. Francisco Edson Lucena Feitosa ${ }^{1,2}$. Raquel Autran Coelho ${ }^{1,2}$.

1 Universidade Federal do Ceará (UFC), Fortaleza, Ceará, Brasil. 2 Maternidade Escola Assis Chateaubriand (MEAC), Fortaleza, Ceará, Brasil.

\section{RESUMO}

Introdução: a doença trofoblástica gestacional (DTG) compreende um grupo heterogêneo de proliferação celular originada a partir do epitélio trofoblástico, dividindo-se em mola hidatiforme completa ou parcial e em neoplasias trofoblásticas gestacionais. A mola hidatiforme ocorre em torno de 1:200-400 gestações no Brasil. Objetivo: avaliar fatores clínicos e epidemiológicos relacionados aos achados histopatológicos de DTG entre mulheres do estado do Ceará, Brasil. Metodologia: pesquisa quantitativa, descritiva, transversal, do tipo retrospectivo, por meio de revisão de prontuários, totalizando 203 pacientes com diagnóstico histopatológico confirmado entre 2012 e 2015. Avaliou-se dados clínico-epidemiológicos e laboratoriais para os casos de mola hidatiforme completa (MHC) e de mola hidatiforme parcial (MHP). Resultados: foram identificados 203 casos de doença trofoblástica gestacional, 49,2\% casos de MHC e 49,7\% de MHP. Não houve diferença estatisticamente significante para idade, paridade, recidiva do sangramento, presença de hipertensão, tipo de sangramento à internação, eliminação de vesículas, cistos tecaluteínicos de ovário e evolução para neoplasias trofoblásticas gestacionais (NTG) entre mulheres com MHC e MHP. A presença de sangramento no momento da internação foi observada em 76,9\% nos casos de MHC e em 85,5\% nos de MHP. 14,3\% dos casos de MHC e 12,1\% dos casos de MHP evoluíram para NTG. Conclusão: não houve diferença estatisticamente significante entre fatores clínico-epidemiológicos para mulheres com MHC e MHP.

Palavras-chave: Doença trofoblástica gestacional. Gonadotropina coriônica. Mola hidatiforme.

\begin{abstract}
Introduction: Gestational trophoblastic disease (DTG) comprises a heterogeneous group of cell proliferation originating from the trophoblastic epithelium. It is divided into complete, partial, and gestational trophoblastic neoplasms. The hydatidiform mole occurs around 1:200-400 gestations in Brazil. Objective: To emphasize the clinical factors related to the histopathological findings of GFR among women from the states of Ceará, Brazil. Methods: A retrospective quantitative, descriptive, cross-sectional study was performed by means of medical records, totaling 203 patients with confirmed histopathological diagnosis between 2012 and 2015. Clinical-epidemiological and laboratory data were evaluated for cases of complete hydatidiform mole (MHC) and partial hydatidiform mole (MHP). Results: 203 cases of gestational trophoblastic disease, $49.2 \%$ cases of MHC and 49.7\% of MHP were identified. There was no statistically significant difference for age, parity, recurrence of bleeding, presence of hypertension, type of bleeding at admission, vesicle elimination, ovarian cytorutinic cysts and evolution for gestational trophoblastic neoplasia (GTN) among women with MHC and MHP. The presence of bleeding at the time of hospitalization was observed in $76.9 \%$ in MHC cases and $85.5 \%$ in MHP cases. $14.3 \%$ of MHC cases and $12.1 \%$ of MHP cases progressed to NTG. Conclusion: There was no statistically significant difference between clinical and epidemiological factors for women with MHC and MHP.
\end{abstract}

Keywords: Gestational trophoblastic disease. Chorionic gonadotropin. Hydatidiform mole.

Autor correspondente: Raquel Autran Coelho, Rua Silva Jatahy, número 1245, Meireles, Fortaleza, Ceará. CEP: 60165-070. Telefone: +55 85 99921-7664. E-mail: raquelautrancp@gmail.com

Conflito de interesses: Não há qualquer conflito de interesses por parte de qualquer um dos autores.

Recebido em: 18 Nov 2018; Revisado em: 18 Fev 2019; Aceito em: 22 Fev 2019. 


\section{INTRODUÇÃO}

A doença trofoblástica gestacional (DTG) compreende um grupo heterogêneo de proliferação celular do epitélio trofoblástico. Divide-se em formas clínicas benignas: mola hidatiforme completa (MHC) e parcial (MHP), e malignas: neoplasias trofoblásticas gestacionais (NTG), representadas pela mola invasora, coriocarcinoma e tumor do sítio placentário. ${ }^{1,2}$ Possuem como marcador a dosagem de Gonadotrofina Coriônica Humana (hCG). A mola hidatiforme, sua forma mais comum, ocorre em torno de 1:200-400 gestações no Brasil, cerca de 5 a 10 vezes mais frequente que na Europa e América do Norte. ${ }^{1}$

Mulheres com DTG classicamente apresentam como sintoma mais frequente o sangramento transvaginal, podendo manifestar também hiperêmese gravídica, pré-eclâmpsia precoce, útero aumentado para a idade gestacional, hipertireoidismo e cistos teca-luteínicos ovarianos. O hCG mostra-se aumentado, frequentemente não compatível com a idade gestacional. Observa-se tendência ao diagnóstico cada vez mais precoce da DTG, diminuindo a frequência de sintomas clínicos associados. ${ }^{1}$ Não houve, contudo, diminuição do risco de evolução para $\mathrm{NTG}^{3,4} \mathrm{O}$ maior estudo epidemiológico conduzido no Brasil sobre a doença trofoblástica constatou que a NTG se desenvolveu em 24,6\% dos pacientes que apresentavam MHC e em 7,6\% dos casos de MHP. ${ }^{5}$

A MHC resulta da fecundação de um óvulo com núcleo ausente ou inativo por um espermatozoide $23 \mathrm{X}$ ou por dois espermatozoides, originando uma célula $46 \mathrm{XX}$ ou 46 $\mathrm{XX} / 46 \mathrm{XY}$, respectivamente. Não há elementos fetais. Já o cariótipo da MHP é triploide, 69 XXY, 69 XXX ou tetraploide, 92 XXXY, resultado da fertilização de um óvulo normal por dois espermatozoides ou um espermatozoide diploide. Pode haver feto, porém é inviável e apresenta crescimento intrauterino restrito e múltiplas malformações. ${ }^{6}$ Ao exame histológico, a MHC exibe anormalidades que envolvem a totalidade ou a maior parte do tecido viloso. As vilosidades coriônicas estão aumentadas, apresentando cavitação central (cisternas) e não possuem vasos adequadamente desenvolvidos. A característica mais marcante é a extensiva proliferação trofoblástica, que envolve toda a circunferência dos vilos. A MHP, por sua vez, demonstra aumento dos vilos e perturbação da arquitetura em apenas uma parte deles. ${ }^{7}$

Quando as características morfológicas das molas estão bem desenvolvidas, o diagnóstico histológico não oferece dificuldades. Apesar da existência de critérios teóricos bem estabelecidos, há grande variação intra e interobservadores para o diagnóstico morfológico das molas, especialmente nas suas formas mais precoces. Estima-se que 50\% dos verdadeiros MHPs não podem ser diagnosticados com precisão pela rotina histologia devido à falta de características histológicas específicas. ${ }^{7,8}$

O exame histopatológico continua sendo o padrão ouro diagnóstico e atualmente pode-se utilizar como complementação diagnóstica a imunocoloração para p57, um inibidor do ciclo celular. O gene p57KIP2 é transcrito pela mãe, mas o imprinting é determinado pelo pai, não ocorrendo expressão da proteína p57 nas molas completas. ${ }^{7}$

A detecção precoce da forma maligna, seguida do tratamento quimioterápico, promove a cura da doença em mais de $90 \%$ dos casos, ${ }^{2}$ justificando a necessidade do seguimento pósmolar com dosagens seriadas de hCG..$^{1-4}$ A FIGO (Federação Internacional de Ginecologia e Obstetrícia) estabelece os seguintes critérios para o diagnóstico de NTG: platô da hCG sérica em 3 semanas de seguimento ou mais; ascensão de mais de $10 \%$ do valor da hCG em duas semanas de seguimento ou mais; persistência da hCG sérica detectável por 6 meses ou mais após o esvaziamento uterino e diagnóstico histológico de coriocarcinoma. ${ }^{3,9}$

Há poucos dados sobre o diagnóstico de MHP e MHC, bem como de NTG no Brasil, especialmente na região nordeste. Devido à baixa frequência desta doença, os tratamentos atuais são baseados em estudos retrospectivos e pequenas séries de casos. ${ }^{10}$ Essa escassez de informações motivou à busca por avaliação de fatores clínico-epidemiológicos e laboratoriais para classificação histológica de DTG entre mulheres cearenses.

\section{MÉTODOS}

Foi realizado estudo quantitativo, descritivo, transversal, do tipo retrospectivo, por meio de revisão de prontuários. A população do estudo consiste em mulheres atendidas no Serviço de DTG da Maternidade Escola Assis Chateaubriand - Universidade Federal do Ceará (MEAC/UFC), hospital de referência para esses casos no estado do Ceará. Todas tiveram diagnóstico histopatológico de DTG confirmado entre os meses de janeiro de 2012 e dezembro de 2015 .

Foram avaliados fatores clínico-epidemiológicos e laboratoriais para classificação histológica de DTG como: idade, paridade, quadro clínico, nível de hCG, achados ultrassonográficos e evolução da doença.

Foram excluídas mulheres imunossuprimidas e em tratamento quimioterápico ou radioterápico prévio ao diagnóstico.

Para estudo das variáveis foi organizada planilha de Excel da qual os dados foram exportados para o programa Prism 5.0, onde foram aplicados testes do quiquadrado e exato de Fisher para dados consolidados em tabela de contigência, bem como teste t de Student para variáveis independentes.

Este estudo foi aprovado pelo Comitê de Ética em Pesquisa da UFC.

\section{RESULTADOS}

No período entre 2012 e 2015, foram identificados 203 casos de DTG provenientes da MEAC/UFC, sendo 100 de molas completas $(49,2 \%), 101$ parciais $(49,7 \%)$ e 2 de molas invasivas $(0,98 \%)$. 
A média de idade das mulheres foi de 23,8 $( \pm 8,3)$, com paridade de $2,3( \pm 1,8)$ e média de idade gestacional ao diagnóstico de 7,7 semanas $( \pm 7) .8,4 \%$ das pacientes tinha idade menor ou igual a $15,77,8 \%$ tinham idade entre 16 a 34 , e $13,8 \%$ tinham idade acima de 34 anos. Segundo a dosagem inicial de beta hCG, 67,3\% das mulheres tinham níveis inferiores a 50.000 . Somente $1,2 \%$ delas tinham história prévia de doença molar. Apesar de ser o sintoma mais frequente, $46,8 \%$ tinham sangramento leve ao diagnóstico, e $21,5 \%$ não relatavam sangramento uterino. Vale ainda ressaltar que $85,5 \%$ não apresentavam vesículas ao internamento.

Não houve diferença estatisticamente significante para idade, número de gestações e partos ou abortos, para o resultado HP de MHP ou MHC (Tabela 1).

Quanto aos fatores clínicos, não houve diferença significante quanto a recidiva do sangramento, presença de hipertensão, tipo de sangramento na internação, eliminação de vesículas, edema de membros inferiores, sinais de tireotoxicose, cistos tecaluteínicos de ovário ou evolução para NTG, entre pacientes com MHP e MHC (Tabela 2).

Dentre as mulheres que apresentaram evolução a NTG $(8 \%$ das MHC e 7,9\% das MHP), não houve diferença entre as idades para 28,5 e 28,8 anos, respectivamente.

Tabela 1. Classificação histopatológica da DTG de acordo com fatores clínico-epidemiológicos.

\begin{tabular}{llll}
\hline Característica & Classificação & Média & p \\
\hline Idade & MHC & 23,2 & 0,43 \\
& MHP & 24,3 & 0,63 \\
Número de gestações & MHC & 2,4 & 0,67 \\
Número de partos prévios & MHP & 2,2 & 0,68 \\
Número de abortos prévios & MHC & 1,0 & 0,9 \\
\end{tabular}

ANOVA; MHC- Mola Hidatiforme Completa; MHP- Mola Hidatiforme Parcial.

Tabela 2. Classificação da DTG de acordo com desfechos clínicos.

\begin{tabular}{|c|c|c|c|c|}
\hline Quadro clínico das pacientes & & $\operatorname{MHC}(\mathbf{N} / \%)$ & MHP (N/\%) & (1) \\
\hline \multirow[t]{2}{*}{ Recidiva do sangramento } & $\operatorname{Sim}$ & $34(41,5 \%)$ & $36(43,4 \%)$ & \\
\hline & Não & $48(58,5 \%)$ & $47(56,6 \%)$ & 0,06 \\
\hline \multirow[t]{2}{*}{ Presença de hipertensão } & $\operatorname{Sim}$ & $9(11,4 \%)$ & $5(6,1 \%)$ & \\
\hline & Não & $70(88,6 \%)$ & $77(93,9 \%)$ & 1,42 \\
\hline \multirow[t]{4}{*}{ Tipo de sangramento na internação } & Ausente & $18(23,1 \%)$ & $14(15,5 \%)$ & \\
\hline & Leve & $39(50 \%)$ & $35(43,8 \%)$ & \\
\hline & Moderado & $11(14,1 \%)$ & $25(31,3 \%)$ & \\
\hline & Intenso & $10(12,8 \%)$ & $6(7,5 \%)$ & 7,136 \\
\hline \multirow[t]{2}{*}{ Eliminação de vesículas à internação } & Ausente & $9(15,8 \%)$ & $8(13,3 \%)$ & \\
\hline & Presente & $48(84,2 \%)$ & $52(86,7 \%)$ & 0,142 \\
\hline \multirow[t]{2}{*}{ Edema de membros inferiores } & Ausente & $1(1,3 \%)$ & $4(5,3 \%)$ & \\
\hline & Presente & $76(98,7 \%)$ & $71(94,7 \%)$ & 1,944 \\
\hline \multirow[t]{2}{*}{ Sinais de tireotoxicose } & Ausentes & $82(100 \%)$ & $80(98,8 \%)$ & \\
\hline & Presentes & $0(0 \%)$ & $1(1,2 \%)$ & 1,019 \\
\hline \multirow[t]{3}{*}{ Cistos tecaluteínicos de ovário } & Ausentes & $54(80,6 \%)$ & $50(79,4 \%)$ & \\
\hline & Unilaterais & $1(1,5 \%)$ & $2(3,2 \%)$ & \\
\hline & Bilaterais & $12(17,9 \%)$ & $11(17,5 \%)$ & 0,408 \\
\hline \multirow[t]{2}{*}{ Evolução para NTG } & Sim & $8(14,3 \%)$ & $8(12,1 \%)$ & \\
\hline & Não & $48(85,7 \%)$ & $58(87,9 \%)$ & 0,125 \\
\hline
\end{tabular}

Teste Qui-Quadrado; MHC- Mola Hidatiforme Completa; MHP- Mola Hidatiforme Parcial; N: número de mulheres. 
Quando se comparou a idade média entre mulheres que progrediram ou não para NTG, independentemente do histopatológico inicial (MHC ou MHP), observou-se 28,6 e 23,6, respectivamente, sem diferença estatisticamente significante $(p=0,3)$. Também não houve associação da evolução para neoplasia quanto ao número de partos prévios neste estudo.

\section{DISCUSSÃO}

A doença trofoblástica gestacional é um espectro de doenças inter-relacionadas que podem progredir para neoplasia, entre as quais a MHC é a forma mais comum. Apesar do fato de que as MHCs são na sua maioria benignas, o risco de desenvolver neoplasia pode chegar até $18-19 \%{ }^{11}$ Não houve diferença quanto a evolução para NTG entre mulheres com MHC e MHP neste estudo.

O quadro clínico clássico da MHC é mais exuberante, com aumento desproporcional do volume uterino, hipertensão, hiperêmese, edema, eliminação de vesículas e sinais de tireotoxicose. Em relação à evolução de MHC para NTG, a literatura apresenta taxa de evolução entre $15 \%$ a $20 \%{ }^{1,12}$ Pacientes com MHC com pelo menos uma das seguintes características são classificados como de alto risco: 1) Coriônica humana sérica gonadotrofina (hCG) antes de ser evacuado superior a $100.000 \mathrm{UI} / \mathrm{L}$. 2) Tamanho do útero aumentado. 3) Cisto teca-luteínico com diâmetro maior que $6 \mathrm{~cm}$. 4) A idade materna é de pelo menos 40 anos. Mulheres com mais de 40 anos tiveram uma probabilidade de 23$37 \%$ de desenvolver neoplasia persistente após a evacuação uterina, enquanto a probabilidade em mulheres com mais de 50 anos de idade foi tão alto quanto $31-56 \%$. Em comparação, as pacientes relativamente mais jovens tinham menor probabilidade, de $15-20 \% .^{13}$

No presente estudo, não houve diferença estatisticamente significante entre idade, paridade, recidiva do sangramento, presença de hipertensão, tipo de sangramento à internação, eliminação de vesículas, edema de membros inferiores, sinais de tireotoxicose, cistos tecaluteínicos de ovário. Tal fato pode ser justificado pelo diagnóstico precoce da doença, diminuindo sua expressão clínica e presença de complicações. Do ponto de vista clínico, complicações como hiperêmese, pré-eclâmpsia e cistos tecaluteínicos são mais frequentes entre as portadoras de MHC. As pacientes com MHP geralmente apresentam sintomas consistentes com abortamento incompleto ou retido e por isto quase sempre o diagnóstico de MHP é obtido após avaliação histológica de material de curetagem. Estudo prévio observou diferenças notáveis quanto aos níveis de gonadotrofinas e, principalmente, para a taxa de evolução para doença persistente, que é de 18 a $28 \%$ contra 1 a $4 \%$ entre as MHP. $^{14}$

\section{REFERÊNCIAS}

1. Braga A, Obeica B, Moraes V, Silva EP, Amim-Junior J, RezendeFilho J. Doença trofoblástica gestacional - atualização. Revista HUPE. 2014;13(3):54-60.
Zhao et al (2017) encontraram media de idade de 46,9 anos em mulheres com MHC, com paridade media 1,2, e idade gestacional média do diagnóstico de 9,5 semanas. Dentre as manifestações clínicas, $90,1 \%$ se apresentaram com sangramento uterino anormal. Como mostrado nesse estudo, a minoria das mulheres com MHC apresentavam toxemia, hipertireoidismo ou coagulopatia, provavelmente devido ao diagnóstico ultrassonográfico precoce..$^{15}$

Espera-se uma taxa maior de MHP do que MHC, diferentemente de nossos achados. Na apresentação clínica da MHP, mais de $90 \%$ dos casos apresentam-se como abortamentos comuns. ${ }^{12}$ Para justificar a incidência semelhante em nosso serviço, podemos supor que casos de MHP tenham sido confundidos com abortamento comum, não sendo enviados para histopatológico. Tal hipótese reforça a importância de análise histopatológica em todos os produtos da concepção após a evacuação para excluir a mola hidatiforme. Além disso, por tratar-se de hospital de referência, pode haver maior drenagem dos casos de MHC, permanecendo a maioria dos casos de MHP nos serviços de origem, possivelmente por falta de diagnóstico histopatológico. Evidencia-se então a importância do envio para análise de todos os casos de "abortamentos", havendo ou não suspeita de DTG, permitindo que as pacientes tenham diagnóstico e seguimento adequado com dosagem de beta-HCG e evitando complicações futuras, como as formas invasivas e metastáticas.

A limitação deste estudo reside em seu aspecto retrospectivo. Além disso, o número limitado de participantes com evolução para NTG pode afetar os resultados. Os pontos fortes do nosso estudo são o número relevante de pacientes frente à escassez de dados regionais, e que avaliamos também a evolução à NTG entre as mulheres avaliadas. Outro aspecto positivo foi a revisão de lâminas, tendo cada amostra histopatológica sido avaliada por pelo menos dois patologistas independentes.

A epidemiologia das DTGs ainda não está bem estabelecida, com estatísticas longe de serem precisas, devido a problemas de acurácia diagnóstica, critérios de inclusão em coortes de estudo, coleta de dados e interpretação na literatura mundial. Outros estudos controlados multicêntricos, preferivelmente com emprego de técnicas imunohistoquímicas, poderiam preencher lacunas para a melhor compreensão da história natural dessa patologia.

\section{CONCLUSÃO}

Não houve diferença estatisticamente significante entre idade, paridade, recidiva do sangramento, presença de hipertensão, tipo de sangramento à internação, eliminação de vesículas, cistos tecaluteínicos de ovário e evolução para NTG entre mulheres com mola hidatiforme completa e mola hidatiforme parcial.

2. Maestá I, Braga A. Desafios do tratamento de pacientes com doença trofoblástica gestacional. Rev Bras Ginecol Obstet. 2012;34(4):143-6. 
3. Sun SY, Melamed A, Goldstein DP, Bernstein MR, Horowitz NS, Moron AF, et al. Changing presentation of complete hydatidiform mole at the New England Trophoblastic Disease Center over the past three decades: does early diagnosis alter risk for gestational trophoblastic neoplasia? Gynecol Oncol. 2015;138(1):46-9.

4. FIGO Oncology Committee. FIGO staging for gestational trophoblastic neoplasia 2000. Int J Gynaecol Obstet. 2002;77(3):2857.

5. Braga A, Uberti EM, Fajardo MC, Viggiano M, Sun SY, Grillo $\mathrm{BM}$, et al. Epidemiological report on the treatment of patients with gestational trophoblastic disease in 10 Brazilian referral centers. Results after 12 years since International FIGO 2000 consensus. J Reprod Med. 2014;59(5-6):241-7.

6. Shih IM, Mazur MT, Kurman RJ. Blaustein's pathology of the female genital tract. 6. ed. New York: Springer Verlag; 2012. Capítulo 20, Gestational trophoblastic tumors and related tumor-like lesions; p. $1075-1135$.

7. Abbas AK, Fausto N, Kumar V. Robbins \& Cotran Patologia: bases patológicas das doenças. 8. ed. Rio de Janeiro: Guanabara Koogan; 2010. Capítulo 22, O trato genital feminino; p.1065-71.

8. McConnell TG, Murphy KM, Hafez M, Vang R, Ronnett BM. Diagnosis and subclassification of hydatiform moles using p57 immunohistochemistry and molecular genotyping: validation and prospective analysis in routine and consultation practice settings whith development of an algorithmic approach. Am J Surg Pathol. 2009;33(6):805-17.

9. Maestá I, Berkowitz RS, Goldstein DP, Bernstein MR, Ramírez
RA, Horowitz NS. Relationship between race and clinical characteristics, extent of disease, and response to chemotherapy in patients with low-risk gestational trophoblastic neoplasia. Gynecol Oncol. 2015;138(1):50-4.

10. Biscaro A, Braga A, Berkowitz RS. Diagnosis, classification and treatment of gestational trophoblastic neoplasia. Rev Bras Ginecol Obstet. 2015;37(1):42-51.

11. Sun SY, Melamed A, Joseph NT, Gockley AA, Goldstein DP, Bernstein MR, et al. Clinical presentation of complete Hydatidiform mole and partial Hydatidiform mole at a regional Trophoblastic Disease Center in the United States over the past 2 decades. Int J Gynecol Cancer. 2016;26(2):367-70.

12. Lurain JR Gestational trophoblastic disease I: epidemiology, pathology, clinical presentation and diagnosis of gestational trophoblastic disease, and management of hydatidiform mole. Am J Obstet Gynecol. 2010;203(6):531-9.

13. Savage PM, Sita-Lumsden A, Dickson S, Iyer R, Everard J, Coleman $\mathrm{R}$, et al. The relationship of maternal age to molar pregnancy incidence, risks for chemotherapy and subsequent pregnancy outcome. J Obstet Gynaecol. 2013;33(4):406-11.

14. Andrade JM. Hydatidiform mole and gestational trophoblastic disease. Rev Bras Ginecol Obstet. 2009;31(2):94-101.

15. Zhao P, Chen Q, Lu W. Comparison of different therapeutic strategies for complete hydatidiform mole in women at least 40 years old: a retrospective cohort study. BMC Cancer. 2017;17(1):733.

\section{Como citar:}

Almeida LO, Sousa EM, Ribeiro AC, Cavalcante DI, Feitosa FE, Coelho RA. Mola hidatiforme parcial e completa: características clínicas e histológicas. Rev Med UFC. 2019 out-dez;59(4):46-50. 